\title{
Current research trends comparing percutaneous dilatational tracheostomy and conventional surgical tracheostomy: a systematic review
}

\author{
Basavaraj Walikar, Ankush Pandit*, S. M. Rashinkar, M. V. Watwe, Yashaswini K.
}

Department of ENT, Al Ameen Medical College and Hospital, Athani Road, Vijayapur, Karnataka, India

Received: 11 July 2018

Revised: 17 August 2018

Accepted: 20 August 2018

\section{*Correspondence:}

Dr. Ankush Pandit,

E-mail: pndtankush@yahoo.com

Copyright: (C) the author(s), publisher and licensee Medip Academy. This is an open-access article distributed under the terms of the Creative Commons Attribution Non-Commercial License, which permits unrestricted non-commercial use, distribution, and reproduction in any medium, provided the original work is properly cited.

\begin{abstract}
A review of research trends, followed in tracheostomy studies, are discussed by several otolaryngologists as a comprehensive review on tracheostomy is extremely difficult to compile mainly due to its voluminous nature and the difficulty in obtaining the scattered information, as isolated pieces of research, an attempt has been made to review some of the recent research publications to depict current research trends in the area of tracheostomy. An overview of the literature shows that percutaneous dilatational tracheostomy (PDT) is as safe and effective as surgical tracheostomy (ST). Although early and late post-operative complication rates are not statistically significant in the PDT group, further investigations of long-term outcome following PDT are therefore necessary. Generally, PDT has fewer acute complications than ST, although this may vary by the specific PDT technique. Patient factors also influence complications. In view of the benefit verses risks in tracheostomy, PDT may be considered the procedure of choice for performing elective tracheostomy in critically ill adult patients.
\end{abstract}

Keywords: Surgical tracheostomy, Percutaneous dilatational tracheostomy, Intensive care unit, Operation room, Stoma

\section{INTRODUCTION}

Tracheostomy is one of the most common procedures performed by otolaryngologists. Tracheostomy is usually performed in patients with difficult weaning from mechanical ventilation and is done mostly in critically ill patients, many of whom may hardly survive. Critically ill patients often receive tracheostomy for continued airway support. There are basically two approaches of tracheostomy: open surgical tracheostomy (OST or ST) and percutaneous dilatational tracheostomy (PDT). The traditional or conventional method involves an open surgical technique performed in operating room (OR). In general, more difficult patients who need prolonged treatment are usually given the standard conventional treatment $(\mathrm{ST})$ which require transport from intensive care unit (ICU) to operating theatre (OT) where surgical team performs the open or surgical tracheostomy. Conventional Surgical tracheostomy involves full dissection of pretracheal tissues and insertion of tracheostomy tube into the trachea under direct vision. ${ }^{1}$ More than 300 years have passed since Sanctorious invented percutaneous tracheostomy. Recent modifications of the instruments used made this old technique suitable for modern surgery. Since Seldinger's introduction of his wire guide approach to arterial catheterization, PDT was first described in 1957 and became increasingly popular after the release of commercially available kit in $1985 .^{2-4}$ This technique involves the use of blunt dilatation to open the pretracheal tissue for passage of tracheostomy tube. All percutaneous techniques use the same underlying 
principles with modifications for the particular organs involved and the purpose of the procedure: drainage of cavity, insertion of permanent pace maker electrodes and placement of a tracheostomy. ${ }^{4}$ Since the mid 1980's PDT has been performed by otolaryngologists and nonotolaryngologists with increasing frequency, yet an energized discussion into the literature has raised questions about the superiority of one technique over the other in regard to the peri-operative and long-term postoperative complications. Several prospective studies and meta-analysis reviews have addressed the question and compared ST with PDT in different ways. The main aim of the present review is to summarize some of this literature to highlight the superiority of one technique to other.

\section{LITERATURE REVIEW}

An energized discussion into the literature has raised questions about the superiority of one technique verses the other in regard to the peri-operative and long-term post-operative complications. Several prospective studies and meta-analysis reviews have addressed this question and compared ST with PDT. The review will summarize some of the literature to address the question of which technique is superior with respect to associated complications. It should be noted that the important discussion of medical economics related to tracheostomy is beyond the scope of this brief review. The earlier studies of Hazard et al based on 55 elective procedures in bedside Percutaneous Tracheostomy suggest that PDT is rapid and simple, to leave almost no cosmetic deformity and almost free from infectious complications. ${ }^{5}$

Delaney et al in his research paper comparing PDT with ST in critically ill patients (1,212 belonging to 17 randomized clinical trials RCT's) concluded that PDT is associated with reduced incidence of wound infection as compared to ST in critically ill patients, the latter being performed in OR. ${ }^{6}$ The authors further opined that PDT performed electively in the ICU should be the method of choice for performing tracheostomies in critically ill adult patients. Similar results have been obtained by Youseef et al whose study, based on 64 critically ill patients admitted to ICU, revealed that PDT technique is effective and safe as ST with low incidence of postoperative complications. $^{7}$ Investigators Turkmen et al and Weisshord and Merati also suggest that PDT appears to be a safe alternative to traditional ST but there may be high incidence of asymptomatic tracheal stenosis with PDT. ${ }^{8,9}$ The assumption of superiority of PDT over ST gains further support from the studies of Putensen et al whose study for comparison is based on 14 RCT's tested PDT techniques versus ST in 973 patients. ${ }^{10}$ The authors concluded that PDT technique can be performed faster and reduce the stoma inflammation and infection but are associated with increased technical difficulties when compared to ST. They further opined that among PDT technique multiple dilator tracheostomy (MDT) and single step dilatation tracheostomy (SSDT) were associated with the lowest risks of intra-procedural technical difficulties and bleeding and therefore seem to be preferable PDT technique in critically ill adult patients.

\section{COMPARISON OF ST WITH PDT-AN OVERVIEW}

The decision to place a tracheostomy should be made by considering the balance between benefit verses risks of the procedure. Most of the risks and benefits are not precisely known for any surgical technique and in most clinical situations. ${ }^{11}$ Thus, deciding when and if to perform tracheostomy in any particular patient is an individual decision and should be approached as such. Perhaps the best understood factors that should be taken into account are the acute risks of tracheostomy. Most reported literature compares these two techniques and much of our understanding of risks of tracheostomy is based on this dichotomy. However, according to Durbin these comparisons are often flawed and difficult to make because: ${ }^{11}$

- Patients are not prospectively matched and often randomized to receive one or the other type of procedure. In general, more difficult patients are given the standard treatment (i.e., ST).

- The other factor includes the fact that with any technical procedure the level of experience of surgeon performing the procedure will influence the outcome and risks. It is also difficult to make the comparison of techniques, even if done by the same individuals, since experience (and preference) will be different for each of the treatment arms.

- Another problem in comparison is that the patients cannot be blinded since tracheostomy placement looks different.

- Another concern in literature comparing tracheostomy techniques is that there is a wide variety of different techniques of ST and PDT and from the literature it is often difficult to identify the exact details of techniques that were compared. Thus, bias is common in studies of tracheostomy.

- One of the biggest impediments in understanding differences in risks between ST and PDT is lack of standardization of definition of what is a particular outcome or risk as there are no common standard definition of risks and complications associated with tracheostomy. In addition to "medical complications" some authors report efficiency measures (time it takes to perform the procedure) and cost to compare different techniques. Thus, bias is again common in studies of tracheostomy.

ST, one of the oldest surgical procedures, probably existing for more than 3000 years was standardized by Jackson (c.f. Turkmen et al, 2008). ${ }^{8}$ Though reported to have a complication rate of upto $66 \%$, yet the mortality rate associated with ST itself is very low. However, postoperative complications such as bleeding, cellulitis infection of the stoma and bad cosmetic results still exist 
and are relatively frequent. ${ }^{8}$ The latter authors in their study on 30 patients (15 for PDT and 15 for ST) while comparing the two techniques concluded that PDT is as safe and effective as ST. However, PDT has a lower rate of early infections and complications than ST. Gambale et al also reported low rate of complications in PDT techniques. $^{12}$ Further, tracheal stenosis is a late complication following any trechostomy on long term intubation. The late complications of PDT however have not been extensively studied. Klussmann et al reported a total atresia of the sub-glottic larynx and cervical trachea after PDT. ${ }^{13}$ Turkmen et al also found severe complications of tracheal stenosis (asymptomatic in nature) in 2 out of 30 patients. $^{8}$ The complications of ST seem to be relatively inordinately high in the face of a relatively small surgical procedure of PDT, yet it is still performed in 33 to $50 \%$ of critically ill patients especially in neurological disorders. ${ }^{14,15}$ Elective ST in patients on long term ventilator support is widely accepted procedure in ICU. However, after the advent of Seldinger guidewire technique, PDT has almost replaced ST. Despite, the long-term experience with ST, the technique still has many complications, with an overall incidence of 6-66\%, including pneumothorax or subcutaneous emphysema (4$17 \%)$, tube dislodgement $(0-7 \%)$, local haemorrhage (3$37 \%)$, stomal infection (17-36\%) and a mortality rate of $0-5.3 \% .^{8}$ PDT on the other hand, requires only a small skin incision, minimal blunt dissection of the anterior tracheal structures, takes only 1-10 min to perform and is commonly performed at bedside.

In contrast to ST, some authors have substantiated the reduced incidence of bleeding and stomal infection with PDT because PDT offers smallest possible tube and stoma consistent with adequate air flow and suctioning ability. This minimal size aids in avoiding haemorrhage when properly done, large vessels are avoided and oozing accompanying the small incision is tamponated by the snug fitted to the tube. In addition, the problem of infection is reduced since less tissue is exposed to possible contamination. The procedure is done bedside (ICU) using local anaesthesia with no standby anaesthetist, with the patient most of the time on a ventilator and with a trans-laryngeal tube in place. There is no need for transportation to OR. There are some definite advantages over standard tracheostomy (ST). This assumption also gains further support from the findings of Turkmen et al and Youssef et al. ${ }^{7,8}$ The former in his study opined that PDT was not associated with significant haemorrhage, purulent infection at the stoma or any lethal complication. Griggs et al also found that PDT technique was associated with a shorter procedural time and a significantly fewer morbidity, in comparison to the standard ST technique and this is likely due to the good experience of otolaryngologists in their technique. ${ }^{16}$ The main advantage of PDT is its application in the ICU as a bedside procedure which prevents any unnecessary delays and risks of transfer to the operating room (OR). The percutaneous technique can be used at the cricothyroid level, the sub cricoid level or lower between the first and second tracheal cartilages when there is a question of damage to the larynx by trans-laryngeal intubation, the lower level site is mandatory below the cricoid cartilage. ${ }^{4}$ Percutaneous subcricoid tracheostomy, properly performed and timed, is recommended as the operation of choice except in emergencies, in the presence of enlarged thyroid glands, in morbidly obese and in children. ${ }^{4}$ The authors further suggest a size of 6-8 $\mathrm{mm}$ inner diameter tracheostomy tubes to be used though, non-rigid tubes are strongly recommended.

\section{CONCLUSION}

The success of PDT technique has caused gradual abandoning of surgical procedure in adult ICU patients as it has shown many advantages over ST.

\section{ACKNOWLEDGMENTS}

The authors are highly thankful to Prof. (Dr.) Ashok K. Pandit, Former Director, Centre of Research for Development, University of Kashmir, Srinagar (Jammu and Kashmir) for going through the early draft of the paper and making some necessary corrections.

Funding: No funding sources

Conflict of interest: None declared

Ethical approval: Not required

\section{REFERENCES}

1. McWhorter AJ. Tracheotomy: timing and techniques. Current Opinion in Otolaryngol Head Neck Surg. 2003;11(6):473-9.

2. Seldinger SI. Catheter replacement of the needle in percutaneous arteriography:a new technique. Acta Radiologica. 1953;39(5):368-76.

3. Shelden CH, Pudenz RM, Tichy FY. Percutaneous Tracheotomy. J Am Med. Assoc. 1957;165:206870.

4. Ciaglia P, Firsching R, Syniec C. Elective Percutaneous Dilatational Tracheostomy:A New Simple Bedside Procedure; Preliminary Report. Chest. 1985;87(6):715-9.

5. Hazard PB, Garrett HE, Adams JW, Robbins ET, Aguillard RN. Bedside Percutaneous Tracheostomy: Experience with 55 Elective Procedures. Ann Thorac Surg. 1988;46:63-7.

6. Delaney A, Bagshaw SM, Nalos M. Percutaneous Dilatational Tracheostomy versus surgical Tracheostomy in critically ill Patients: A Systematic Review and meta-analysis. Critical Care. 2006;10(2):1-13.

7. Youssef TF, Ahmed MR, Saber A. Percutaneous dilational versus conventional surgical tracheostomy in intensive care patients. North Am J Med Sci. 2011;3(11):508-12.

8. Turkmen A, Altan A, Turgut N, Yildirim G, Ersoy A, Koksal C, et al. Comparison of Percutaneous 
Dilational Tracheostomy with Surgical Tracheostomy. M E J Aneszth. 2008;19(5):1055-67.

9. Weissbrod PA, Merati AL. Is Percutaneous Dilational Tracheostomy Equivalent to Traditional Open Surgical Tracheostomy with regard to Perioperative and Postoperative Complications? Laryngoscope. 2012;122:1423-4.

10. Putensen C, Theuerkaul N, Guenther U, Vargas M, Pelosi P. Percutaneous and Surgical Tracheostomy in Critically ill Adult Patients: A meta-analysis. Critical Care. 2014;18:1-21.

11. Durbin CJ. Early Complications of Tracheostomy. Respiratory Care. 2005;50(4):511-5.

12. Gambale G, Cancellieri F, Baldini U, Vacchi Suzzi M, Baroncini S, Ferrari F, et al. Ciaglia Percutaneous Dilational Tracheostomy. Early and Late Complications and Follow up. Inerva Anestesiol. 2003;69(11):825-30.

13. Klussmann JP, Brochhagen HG, Stittel C, Eckel HE, Wasserman K. Atresia of the Trachea following
Repeated Percutaneous Dilational Tracheotomy. Chest. 2001;119:961-4.

14. Arabi YM, Alhashemi JA, Tamim HM, Esteban A, Haddad SH, Dawood A, et al. The impact of time to tracheostomy on mechanical ventilation duration, length of stay and mortality in intensive care unit patients. J Crit Care. 2009;24:435-40.

15. Veenith T, Ganeshmoorthy S, Standley T, Carter J. Young P. Intensive care unit tracheostomy:A snapshot of UK practice. Int Arch Med. 2008;1:21.

16. Griggs WM, Myburg JA, Worthley LIG. A prospective comparison of a percutaneous tracheostomy technique with standard surgical tracheostomy. Intensive Care Med. 1991;17:261-3.

Cite this article as: Walikar B, Pandit A, Rashinkar SM, Watwe MV, Yashaswini K. Current research trends comparing percutaneous dilatational tracheostomy and conventional surgical tracheostomy: a systematic review. Int J Otorhinolaryngol Head Neck Surg 2018;4:1121-4. 